\title{
Efficient and Accurate EM Simulation Technique for Analysis and Design of MMICs
}

\author{
Noyan Kınayman and M. I. Aksun \\ Department of Electrical and Electronics Engineering, Bilkent University, 06533 Ankara, Turkey
}

Received 17 January 1996; revised 20 August 1996

\begin{abstract}
A numerically efficient technique for the analysis and design of MMIC circuits is introduced and applied to some realistic problems. The formulation is based on the method of moments (MoM) in the spatial domain, and utilizes closed-form Green's functions. Incorporating the closed-form Green's functions into the MoM has resulted in an efficient and accurate CAD algorithm. This is because use of the closed-form Green's functions not only eliminates the calculation of the spatial-domain Green's functions, but also makes it possible to evaluate the MoM matrix elements analytically We have demonstrated the application of this method here for some stripline and microstrip geometries, and compared the results with those obtained from commercial EM software, em (Sonnet Software, Inc.) ๑ 1997 John Wiley \& Sons, Inc. Int J Microwave Millimeter-Wave CAE 7: 344-358, 1997.
\end{abstract}

Keywords: complex image method; MMIC; monolithic microwave integrated circuits; planar circuits; method of moments

\section{INTRODUCTION}

With the advent of monolithic microwave integrated circuits (MMICs), printed structures in a planar layered medium have gained a lot of interest. They are rugged, easy to produce, and reproducible, which make them attractive for MMICs. In addition, they also have low cross-section and weight, which makes them useful in airborne applications. Thus, development of a rigorous and efficient technique to characterize such structures has become an important issue in computational electromagnetics. Basically, there are two approaches in the characterization of printed structures: (i) approximate but numerically efficient methods, like quasistatic methods [1-3]; and (ii)

Correspondence to: M. I. Aksun

Contract grant sponsor: NATO Scientific Affairs Division

Contract grant sponsor: Scientific and Technical Research Council of Turkey

Contract grant number: EEEAG-132 accurate but computationally expensive methods like the method of moments (MoM) [4], the finite element method [5], and the finite-difference time-domain method [6]. Among these approaches, the spatial-domain and the spectraldomain MoM are the most commonly used numerical techniques for accurate analyses of printed geometries.

In this study, the spatial-domain MoM employing subdomain basis functions is used to solve the mixed potential integral equation (MPIE) [7-9] for current distribution on the conductors, which are immersed in a planar layered medium, as shown in Figure 1.

Using the spatial-domain Green's functions in the MoM for a planar layered medium requires numerical integration of Sommerfeld integrals [10], which is a very time consuming process. But, it was recently recognized that the Sommerfeld integrals can be approximated analytically, which provides the closed-form spatial-domain Green's 


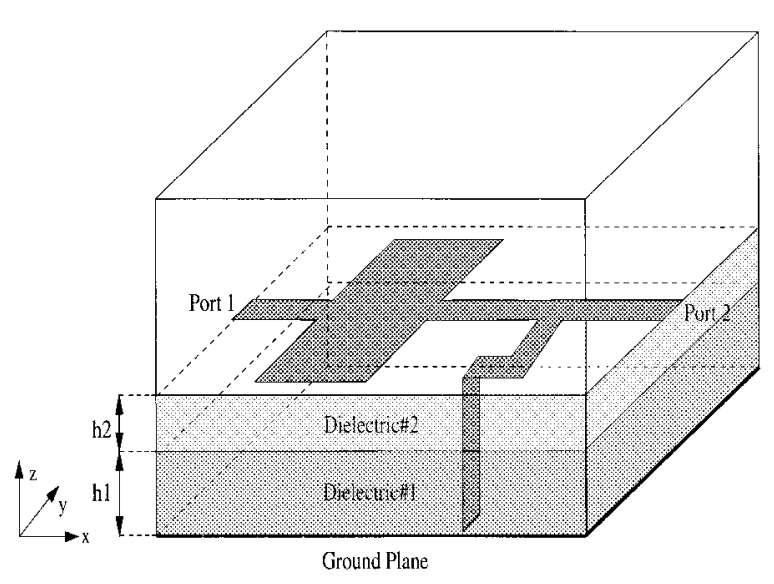

Figure 1. A typical printed geometry.

functions [11]. With the use of these closed-form Green's functions in conjunction with the spatial-domain MoM, the computational efficiency of the MoM is significantly improved for small- and moderate-size geometries. In addition, when the closed-form Green's functions are employed in the MoM, the resulting MoM matrix elements can also be evaluated analytically, which further improves the computational efficiency of the technique [12].

So far, the technique is discussed from the analysis point of view, but, in designing a printed circuit, optimization plays an important role as well. An optimization algorithm, in general, requires the simulation program to analyze the modified geometry at each iteration. Meanwhile, if the simulation program needs to analyze the modified geometry from scratch, the optimization algorithm would be computationally expensive even though the simulation program is efficient. Therefore, to assess the numerical efficiency of a simulation technique it is necessary to consider the suitability of the technique for optimization. The technique that we present here is very suitable because the effects of newly added or removed metallic regions are handled very fast by adding an additional row-column pair to or removing a row-column pair from the original matrix for each additional basis function, respectively. In addition to the efficient filling of the MoM matrix of the modified geometry, the solution time of the modified matrix equation can also be improved by using the solution of the matrix equation in the previous iteration through the method known as the order recursive Gaussian elimination approach [13].
In the next section, application of the spatialdomain MoM to the solution of the MPIE is briefly explained from a theoretical point of view, and the use of the closed-form Green's functions is introduced, without giving the details. Then, assuming that the surface current density is obtained, the de-embedding of the circuit parameters, such as input impedance and S-parameters, is explained. Then, in the fourth section, some practical passive MMIC components are characterized to illustrate the use of the proposed method, and the results are compared with those obtained from commercial EM software, em (Sonnet Software, Inc.). The final section presents the conclusions.

\section{THEORETICAL BACKGROUND}

The geometry of a general printed structure in a multilayer medium is shown in Figure 1. The layers are planar and assumed to be infinitely wide in the horizontal plane, and the conductors are lossless and infinitesimally thin. An $e^{j w t}$ time dependence is assumed and suppressed.

The field components in a planar stratified medium can be expressed as [7]:

$$
\begin{aligned}
E_{x}= & -j w G_{x x}^{A} * J_{x}+\frac{1}{j w} \frac{\partial}{\partial x} \\
& \times\left[G_{x}^{q} * \frac{\partial J_{x}}{\partial x}+G_{y}^{q} * \frac{\partial J_{y}}{\partial y}+G_{z}^{q} * \frac{\partial J_{z}}{\partial z}\right] \\
E_{y}= & -j w G_{y y}^{A} * J_{y}+\frac{1}{j w} \frac{\partial}{\partial y} \\
& \times\left[G_{y}^{q} * \frac{\partial J_{y}}{\partial y}+G_{x}^{q} * \frac{\partial J_{x}}{\partial x}+G_{z}^{q} * \frac{\partial J_{z}}{\partial z}\right] \\
E_{z}= & -j w G_{z x}^{A} * J_{x}-j w G_{z y}^{A} * J_{y} \\
& -j w G_{z z}^{A} * J_{z}+\frac{1}{j w} \frac{\partial}{\partial z}\left[G_{x}^{q} * \frac{\partial J_{x}}{\partial x}\right. \\
& \left.+G_{y}^{q} * \frac{\partial J_{y}}{\partial y}+G_{z}^{q} * \frac{\partial J_{z}}{\partial z}\right]
\end{aligned}
$$

where $G_{x x, y y, z x, z z}^{A}$ and $G_{x, y, z}^{q}$ are the spatialdomain vector and scalar Green's functions, respectively; $J_{x}, J_{y}, J_{z}$ are the surface current densities; and * denotes the convolution operator. Because the traditional form of the Green's functions are employed in the formulation [14], the Green's function of the scalar potential is not unique for HED and VED. Therefore, the scalar 
potentials given in eq. (1)-(3) are $G_{x}^{q_{e}}=G_{y}^{q_{e}}$ for a HED and $G_{z}^{q_{e}}$ for a VED. In the application of the MoM, the surface current densities are expanded in terms of some suitable basis functions, and the resulting field expressions are tested by a suitable class of testing functions. Here we have chosen the rooftop functions as the basis and testing functions on both horizontal and vertical conductors, resulting in Galerkin's MoM. For the sake of illustration, the basis functions used for a typical printed geometry are shown in Figure 2. Hence, the MoM matrix equation is obtained with typical matrix entries as:

$$
\begin{aligned}
& \left\langle T_{x m}, G_{x x}^{A} * J_{x n}\right\rangle \\
& +\frac{1}{w^{2}}\left\langle T_{x m}, \frac{\partial}{\partial x}\left[G_{x}^{q} * \frac{\partial J_{x n}}{\partial x}\right]\right\rangle \\
& \left\langle T_{z m}, G_{z z}^{A} * J_{z n}\right\rangle \\
& +\frac{1}{w^{2}}\left\langle T_{z m}, \frac{\partial}{\partial z}\left[G_{z}^{q} * \frac{\partial J_{z n}}{\partial z}\right]\right\rangle
\end{aligned}
$$

where $T_{x m}, T_{z m}$ and $J_{x n}, J_{z n}$ denote the testing and basis functions, respectively, and $\langle$,$\rangle desig-$ nates the inner product. After solving the matrix equation, the current distributions on the conductors are found and, from the current distribution, the circuit parameters can be extracted.

If the inner-product terms in eqs. (4) and (5) are examined, it is seen that the spatial-domain Green's functions play an important role in the calculation of the inner products. This is because the spatial-domain Green's functions are obtained from the spectral-domain counterparts through an integral transformation, called the Hankel transform or the Sommerfeld integral in electromagnetics [15]. This transformation is given as:

$$
G=\frac{1}{4 \pi} \int_{S I P} d k_{\rho} k_{\rho} H_{0}^{(2)}\left(k_{\rho} \rho\right) \tilde{G}\left(k_{\rho}\right)
$$

where $G$ and $\tilde{G}$ are the Green's functions in the spatial and spectral domains, respectively; $H_{0}^{(2)}$ is the Hankel function of the second kind; and SIP is the Sommerfeld integration path. Because the aim is to eliminate the numerical integration in eq. (6), the spectral-domain Green's function is approximated by complex exponentials, whose Hankel transform can be performed analytically, resulting in closed-form spatial-domain Green's functions $[16,17]$.

The exponential approximation is performed by using the generalized pencil of function method (GPOF) [18] at three steps along the path shown in Figure 3. This is an extension of the two-level approach introduced recently [17], so the details of this approximation scheme are not included here (one may refer to ref. 17). However, we should note that this multilevel approach eliminates the need to extract the quasistatic images

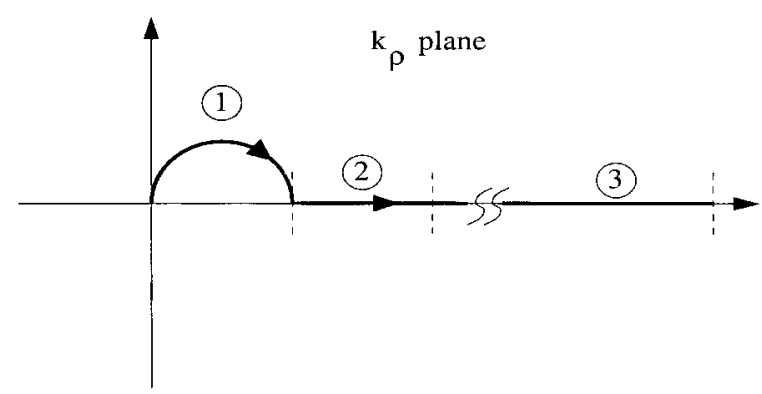

Figure 3. The path used in exponential approximation.

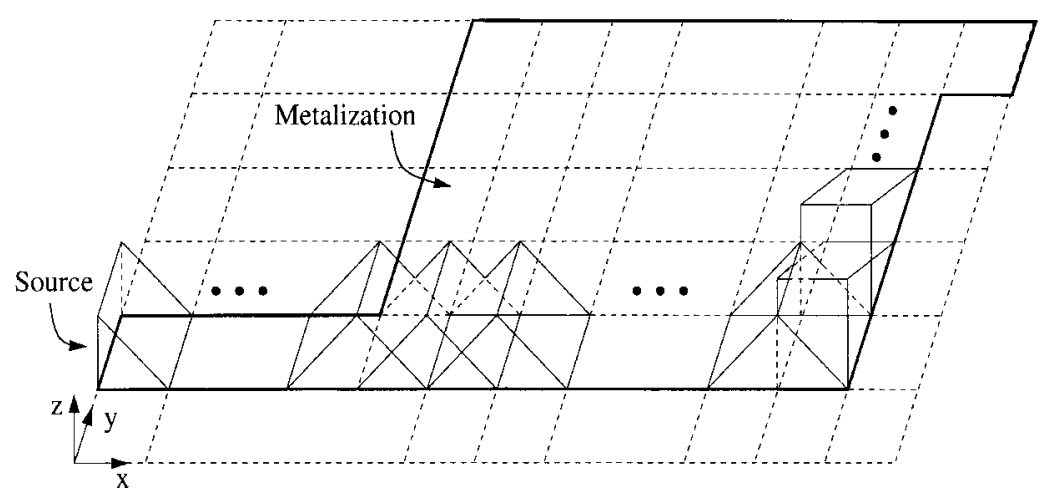

Figure 2. Basis functions used for a typical printed geometry. 
and the surface-wave poles, which further improves the computational efficiency of the derivation of the closed-form Green's functions. The reason for the extension of the two-level approach to the three-level approach can be explained as follows: When a vertical conductor is used, such as shorting pins or via holes, the fields due to the current on this conductor have to be evaluated over a very small distance, $\rho$, requiring a very accurate approximation for large $k_{\rho}$. So, the third region is added onto the two-level approach to give enough emphasis to the approximation for large $k_{\rho}$, without increasing the computational complexity of the whole approximation. As a result of this approximation, the spectral-domain Green's functions are obtained in the following form:

$$
\begin{aligned}
\tilde{G} \cong & \frac{1}{j 2 k_{z}}\left\{\sum_{n=1}^{N_{1}} a_{1 n} e^{-b_{1 n} k_{z}}+\sum_{n=1}^{N_{2}} a_{2 n} e^{-b_{2 n} k_{z}}\right. \\
& \left.+\sum_{n=1}^{N_{3}} a_{3 n} e^{-b_{3 n} k_{z}}\right\}
\end{aligned}
$$

Once the spectral-domain Green's functions are represented as the sum of complex exponentials, each exponential term in eq. (17) can be transformed to the spatial domain via the Sommerfeld identity:

$$
\frac{e^{-j k r}}{r}=\frac{1}{2 j} \int_{S I P} d k_{\rho} k_{\rho} H_{0}^{(2)}\left(k_{\rho} \rho\right) \frac{e^{-j k_{z}|z|}}{k_{z}}
$$

yielding the following Green's function expression in the spatial domain:

$$
\begin{aligned}
G \cong & \sum_{n=1}^{N_{1}} a_{1 n} \frac{e^{-j k_{i} r_{1 n}}}{r_{1 n}}+\sum_{n=1}^{N_{2}} a_{2 n} \frac{e^{-j k_{i} r_{2 n}}}{r_{2 n}} \\
& +\sum_{n=1}^{N_{3}} a_{3 n} \frac{e^{-j k_{i} r_{3 n}}}{r_{3 n}}
\end{aligned}
$$

where $r_{1 n}=\sqrt{\rho^{2}-b_{1 n}^{2}}, \quad r_{2 n}=\sqrt{\rho^{2}-b_{2 n}^{2}}, \quad r_{3 n}$ $=\sqrt{\rho^{2}-b_{3 n}^{2}}$, and $\rho=\sqrt{x^{2}+y^{2}}$. Next, the closed-form representation of the spatial-domain Green's functions are substituted into eqs. (4) and (5) and the resulting integrals, for the convolution and the inner product, are evaluated analytically as described in ref. 12. Hence, the use of the closed-form Green's functions in the MoM resulted in an accurate simulation technique with no numerical integration involved, giving rise to a very efficient and yet accurate EM simulation software.

Although the approach just described seems straightforward, it has some difficulties in cases of geometries with vertical conductors. Because the spectral domain Green's functions are sampled for the purpose of approximation, the variables $z$ and $z^{\prime}$ have to be fixed to the observation and source planes, respectively. Therefore, the resulting closed-form spatial-domain Green's functions become valid only for these specific source and observation points. In cases of only horizontal conductors, this poses no problem because the source and observation planes are already fixed. However, in the case of a vertical conductor, eq. (5) shows that one needs to integrate along $z^{\prime}$ for the convolution integral and along $z$ for the inner-product integral. If the technique just described is used directly, the exponential approximation needs to be performed for every integration point of $z$ and $z^{\prime}$, which would result in an extremely inefficient approach to incorporate a vertical conductor. Instead, the Green's functions for the vertical electric dipole in eq. (5) are written as the inverse Hankel transform of their spectral domain representations, and the integrals on $z$ and $z^{\prime}$ variables are performed analytically in the spectral domain. Then, the resulting functions are transformed to the spatial domain as just described and the remaining integrals are evaluated analytically as before. Note that the spectral-domain Green's functions consist of exponential functions of $z$ and $z^{\prime}$ which permits the analytical integration in the spectral domain [16, 19]. In the approach just described, the number of basis functions selected in the $z$-direction is not limited; therefore, the lengths of the vertical conductors can be arbitrarily long.

\section{METHOD OF DE-EMBEDDING OF PORT DISCONTINUITIES}

Because there exists fringing, reactive, and evanescent fields in the vicinity of source and load terminals, circuit parameters, like input impedances and S-parameters, are obtained by removing these higher order effects from the calculations, which is called de-embedding [20]. The EM simulation technique presented in this work uses impressed current sources (see Fig. 2) to characterize N-port circuits [21].

Because the current densities on the conductors, including the port transmission lines, have 
already been calculated, the current on each port transmission line is written as the linear combination of complex exponentials by using the GPOF as follows:

$$
I(l) \approx \sum_{i=1}^{N} I_{i} \exp \left[\left(\alpha_{i}+j \beta_{i}\right) l\right]
$$

where $l$ is the distance along the port transmission line. If the magnitudes of higher order modes on the port transmission lines are sufficiently small, the current can be expressed by only two exponentials with complex coefficients corresponding to the incident and reflected current waves at the corresponding ports. Then, the Sparameters are calculated from the coefficients of these exponentials and transferred to the desired reference planes [22]. These S-parameters are inherently normalized and referenced to the characteristic impedances of the port transmission lines. Note that, by using this method, the propagation constants on the transmission lines are also found, which enables us to extract the effective permittivity at each frequency of operation. However, in some cases, it might be numerically difficult to extract the propagation constant and the unknown coefficients of the exponentials with sufficient precision from the same current samples by direct application of GPOF. This situation occurs in MMIC structures where the electrical length of the port transmission line is very small. In that case, one can find the propagation constant from a sufficiently long test transmission line which has the same characteristics as the original port transmission line, and then use this propagation constant to fit the current on the original line with complex exponentials through a linear least-squares algorithm [23]. Finally, the S-parameters obtained are converted to the Sparameters with the reference impedance of $50 \Omega$.

For a two-port network, the matrix relation, used to find the S-parameters, can be written in the following form:

$$
\left[\begin{array}{cccc}
I_{11}^{+} & I_{12}^{+} & 0 & 0 \\
0 & 0 & I_{11}^{+} & I_{12}^{+} \\
I_{21}^{+} & I_{22}^{+} & 0 & 0 \\
0 & 0 & I_{21}^{+} & I_{22}^{+}
\end{array}\right]\left[\begin{array}{l}
S_{11} \\
S_{12} \\
S_{21} \\
S_{22}
\end{array}\right]=\left[\begin{array}{l}
-I_{11}^{-} \\
-I_{12}^{-} \\
-I_{21}^{-} \\
-I_{22}^{-}
\end{array}\right]
$$

where $I_{i j}^{+}$and $I_{i j}^{-}$are the current coefficients for incident and reflected waves, respectively, for $i$ th port excitation and $j$ th port observation. Note that, in calculating the S-parameters, it is not necessary to terminate the ports because, both the transmitted and reflected waves are considered in the calculations. To calculate the Sparameters of a general $\mathrm{N}$-port network with characteristic impedances $Z_{0_{i}}$ at port $i$, the following pseudo-code, which fills the matrices in eq. (11), is given here for convenience:

$$
\begin{aligned}
& A \leftarrow 0 \\
& n \leftarrow \text { number of ports } \\
& \text { for } i=0,1,2, \ldots, n-1 \\
& \text { begin } \\
& \quad \text { for } l=0,1,2, \ldots, n-1 \\
& \quad \text { begin } \\
& \quad \text { for } m=0,1,2, \ldots, n-1 \\
& \quad \text { begin } \\
& \quad A_{i * n+m, l+m * n} \leftarrow I_{i l}^{+} * \operatorname{sqrt}\left(Z_{0_{l}}\right) \\
& \quad \text { end } \\
& \quad B_{i * n+l} \leftarrow-I_{i l}^{-} * \operatorname{sqrt}\left(Z_{0_{l}}\right) \\
& \text { end } \\
& \text { end }
\end{aligned}
$$

Note that this derivation of the S-parameters does not require the knowledge of the characteristic impedances of the port transmission lines. However, the need to convert these S-parameters to the S-parameters with a known reference impedance (usually $50 \Omega$ ) makes it necessary to know the characteristic impedances of the port transmission lines. Because a rigorous calculation of the characteristic impedance of a microstrip line in a multilayer medium is quite time consuming, a method based on a quasi-TEM approach is used here, which provides a closed-form expression for the characteristic impedance of a microstrip line and a stripline [24, 25]. This method is valid when the transverse components of the current densities on the port transmission lines are very small as compared with the longitudinal components, which is usually the case for MMICs. Note that the characteristic impedance found from the quasi-TEM approach does not change with frequency, whereas, in reality, it changes with frequency [26, 27]. By using a frequencydependent effective permittivity in the closedform expression for the characteristic impedance, the quasi-TEM approximation is somewhat extended.

\section{EXAMPLES}

In this section, some examples are given and the results are compared with those obtained from em (Sonnet Software, Inc.). One can refer to 


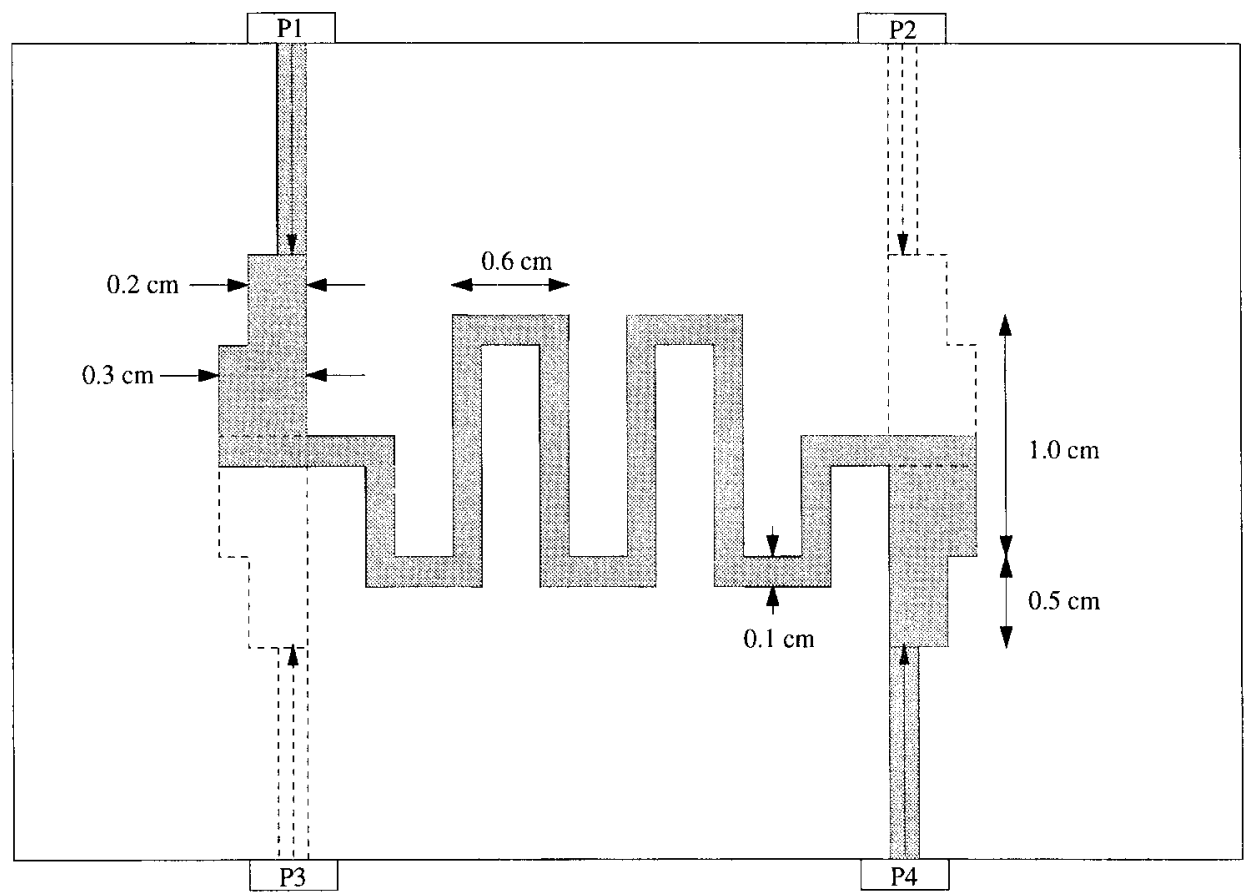

Figure 4. Geometry of the hybrid coupler showing the ports (not to scale).

Carroll and Chang [28] for general information on $\mathrm{em}$ software. The first three examples are the structures which have multilayer metalization, and the last example is a microstrip line with a shunt short-circuited stub to demonstrate the use of the vertical connection. The S-parameters provided here are normalized with respect to $50-\Omega$ reference impedance.

As a first example, a multilayer $3-\mathrm{dB} 90^{\circ}$ hybrid coupler is analyzed. The geometry and layer information of the coupler are given in Figures 4 and 5, respectively. The results obtained from the method presented in this article show very good agreement with the results obtained with $\mathrm{em}$ software (Figs. 6 and 7).
The next example is a coupled-line band-pass filter whose geometry and layer information are given in Figures 8 and 9, respectively. In designing edge-coupled filters, it might be necessary to have small spacings - in other words, large couplings, between the resonant elements, which would make the production of such filters very difficult. This difficulty can be circumvented by implementing such coupled lines in a stripline geometry with broadside coupling $[29,30]$. Here, by using this concept, a third-order band-pass filter is designed by following the design procedure based on the even- and odd-mode impedances of the coupled lines, which are available in the literature [25]. The analysis of this

Ground Plane

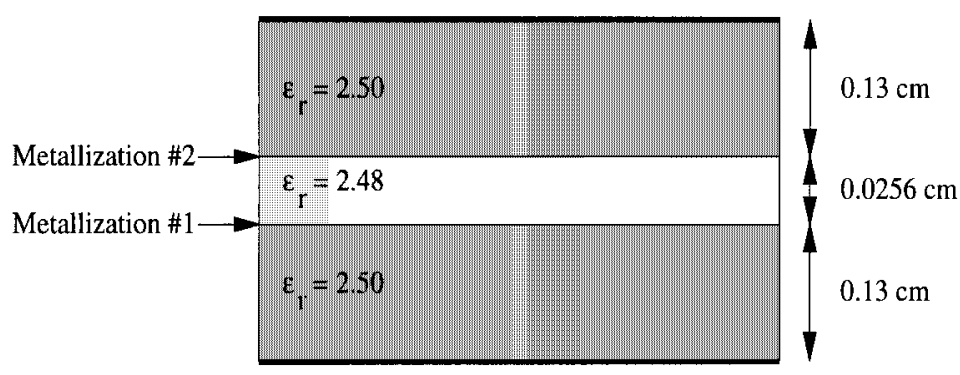

Ground Plane

Figure 5. Layer information for the hybrid coupler shown in Figure 4. 


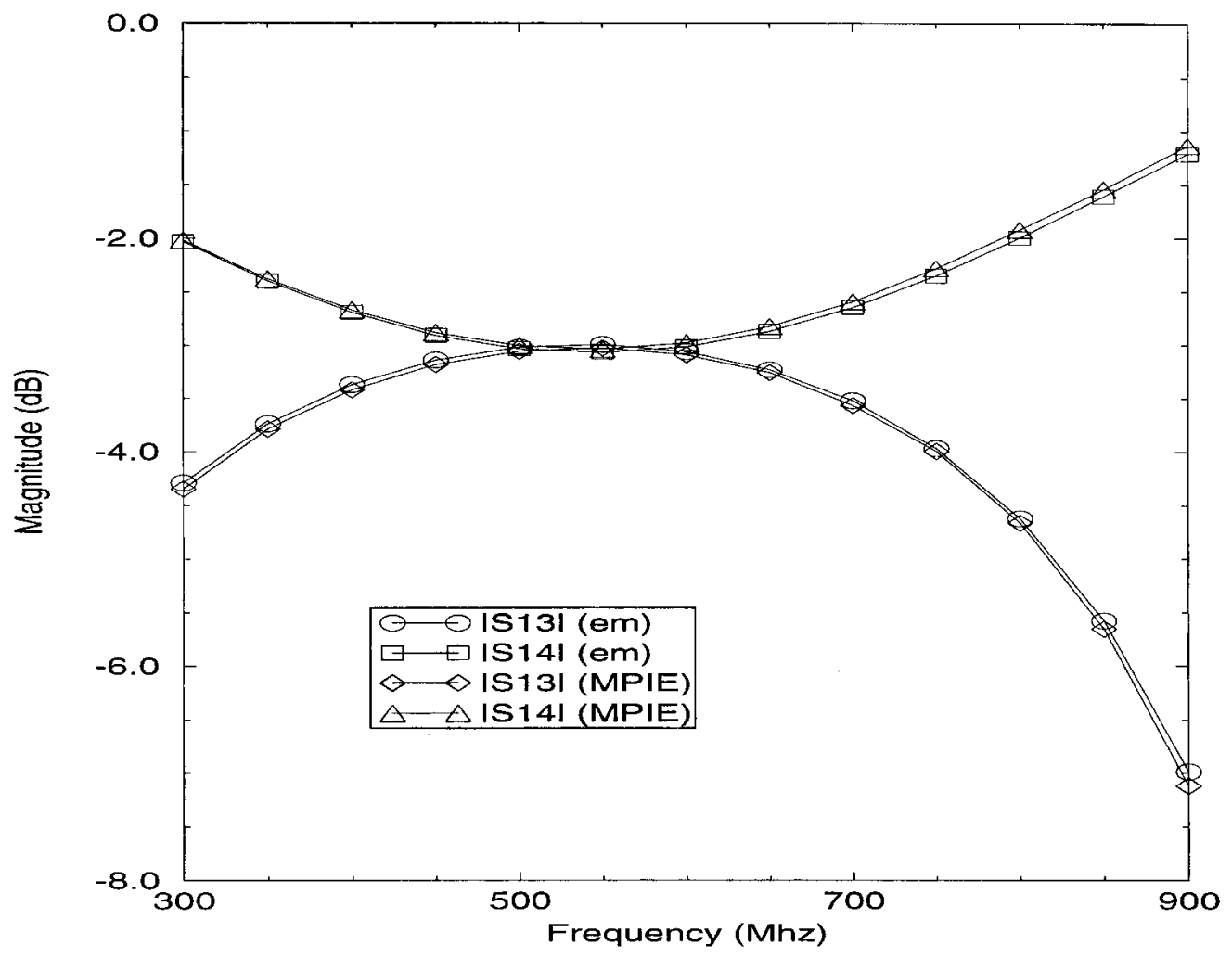

Figure 6. Magnitudes of $S_{13}$ and $S_{14}$ of the hybrid coupler shown in Figure 4.

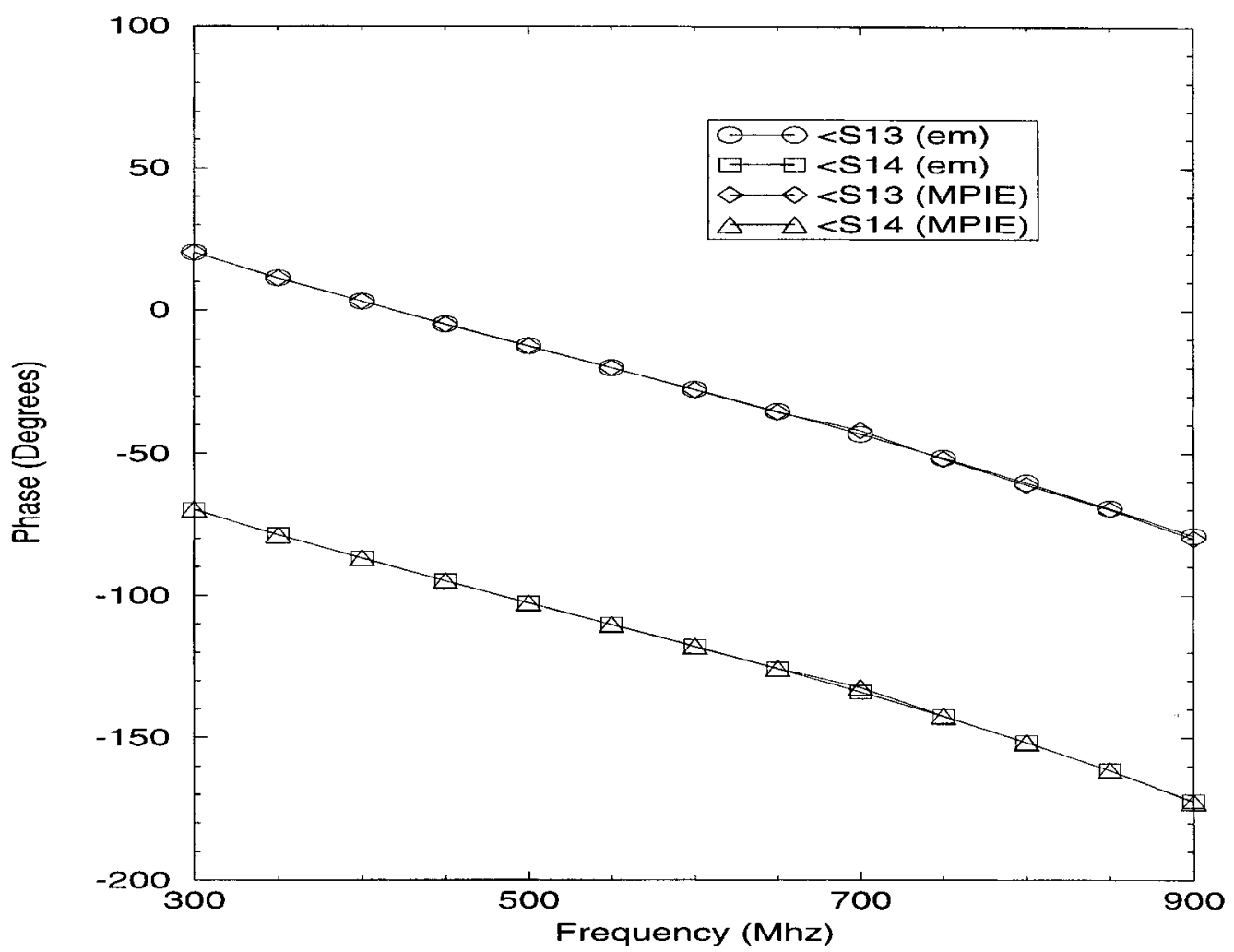

Figure 7. Phases of $S_{13}$ and $S_{14}$ of the hybrid coupler shown in Figure 4. 


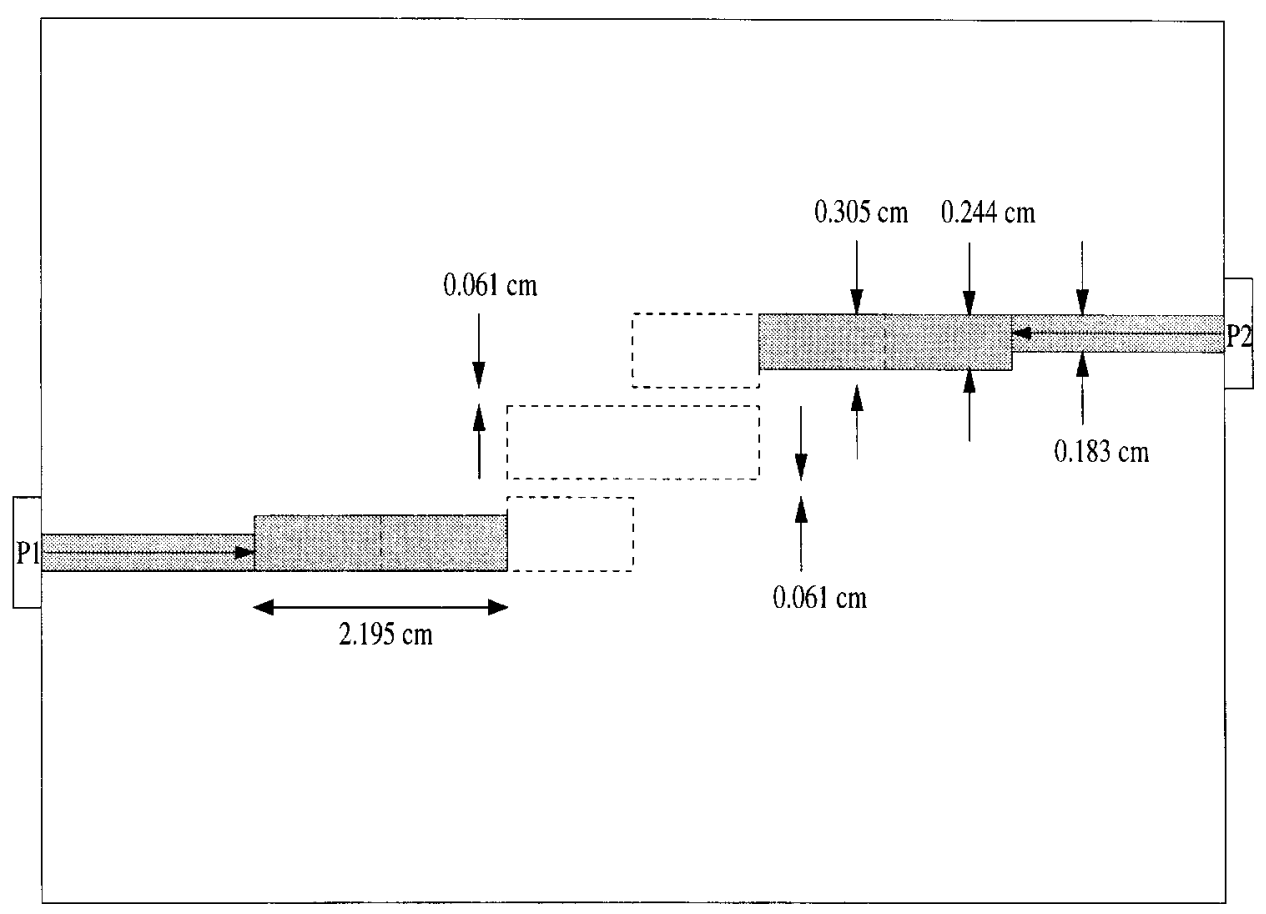

Figure 8. Geometry of the band-pass filter showing the ports (not to scale).

filter is performed by using the method described here and by the em software, and the results are in perfect agreement, as shown in Figure 10 and 11. Note that the response of the filter can be further improved without substantially increasing the computation time by using a fully automated optimization algorithm.

Another example is a proximity-coupled microstrip patch antenna, whose geometry and layer information are given in Figures 12 and 13, respectively. Because the major disadvantage of microstrip antennas is the small bandwidth, this can be improved by using a proximity-coupled feed structure [31], as shown in Figure 12. Note that a tuning stub at the feeding line is used to match the antenna to the characteristic impedance of the line. Here, the antenna is analyzed with and without the tuning stub with reference planes at the stub position and at the antenna edge, respectively, and the results are provided in Figures 14 and 15 . It should be noted that the analysis of a radiating structure by using $\mathrm{em}$ requires some conditions to be satisfied: namely, the size of the box must be increased, the top cover must be

Ground Plane

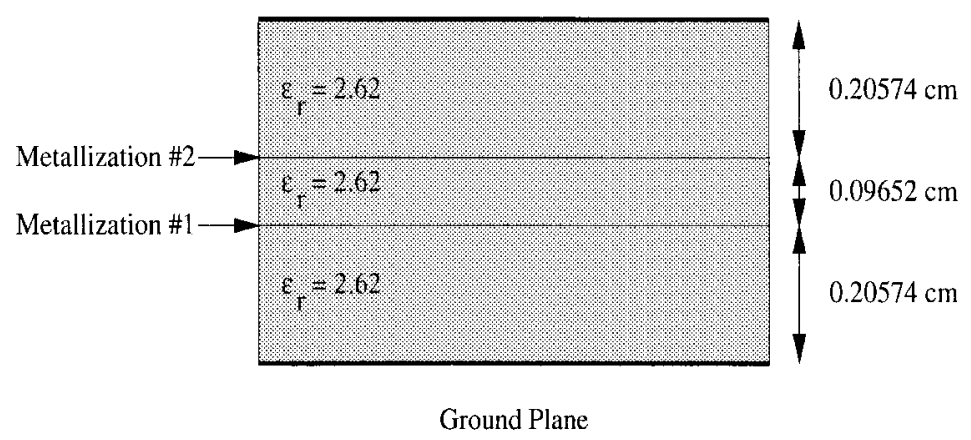

Figure 9. Layer information for the band-pass filter shown in Figure 8. 


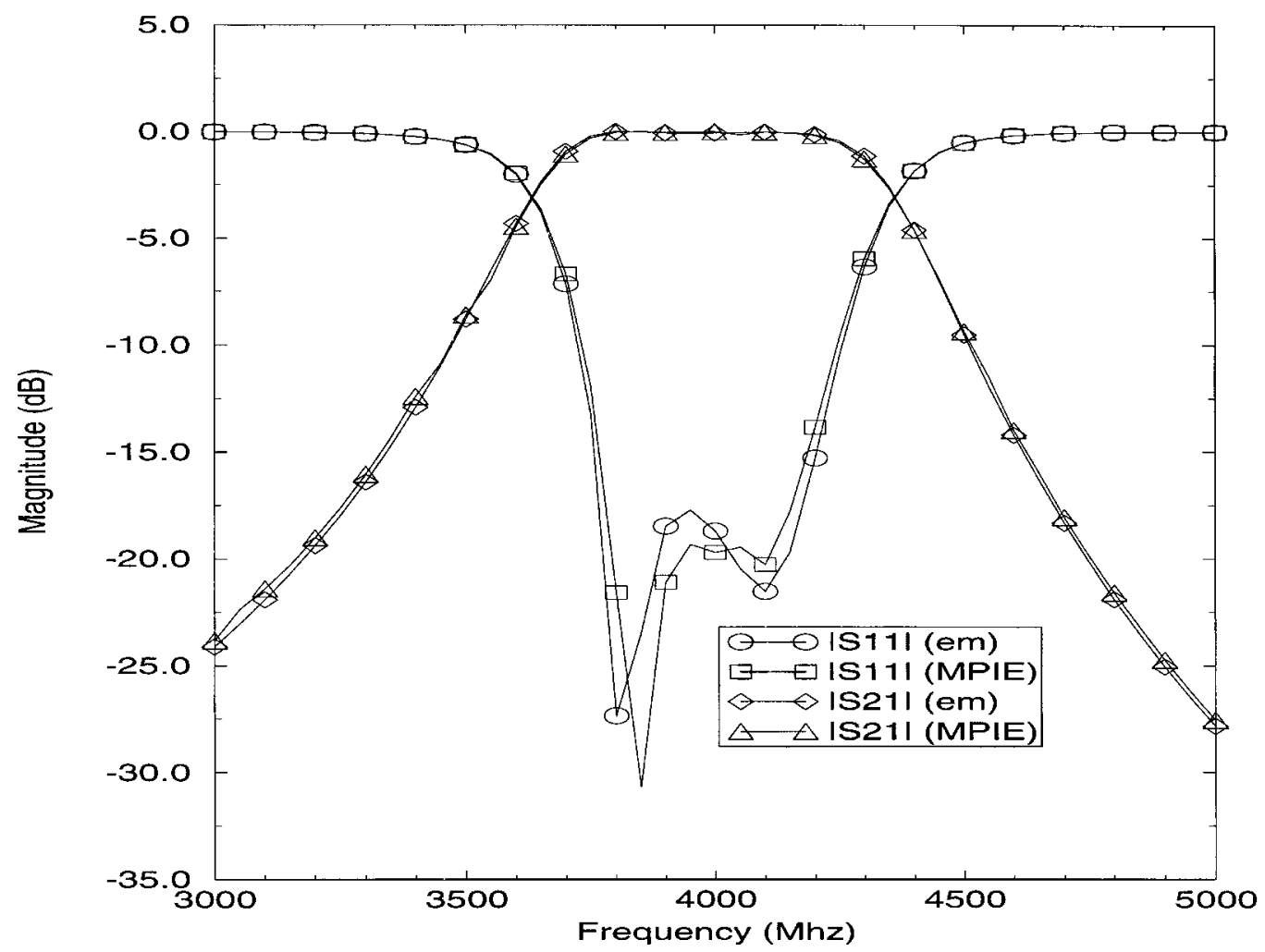

Figure 10. Magnitudes of $S_{11}$ and $S_{21}$ of the band-pass filter shown in Figure 8.

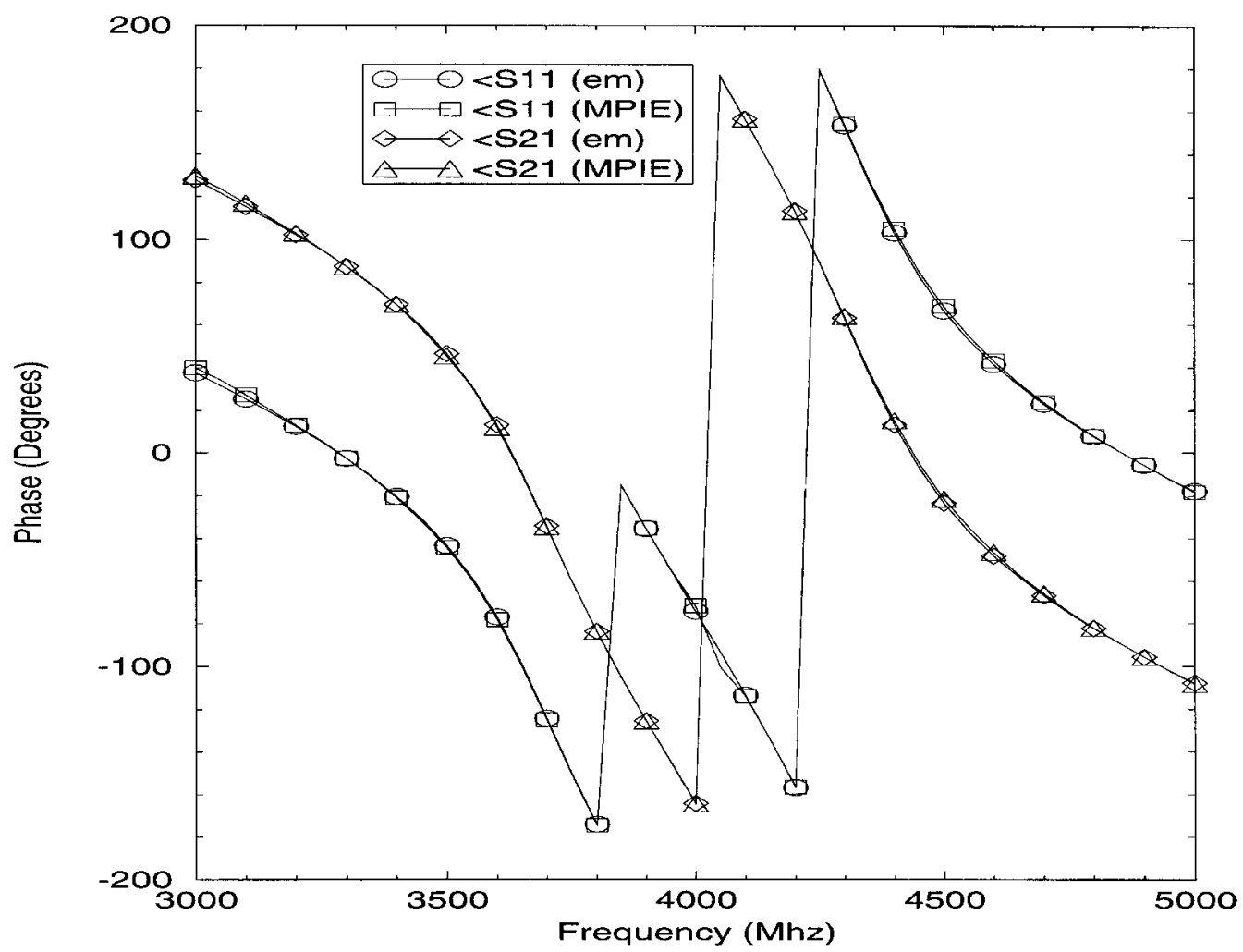

Figure 11. Phases of $S_{11}$ and $S_{21}$ of the band-pass filter shown in Figure 8. 


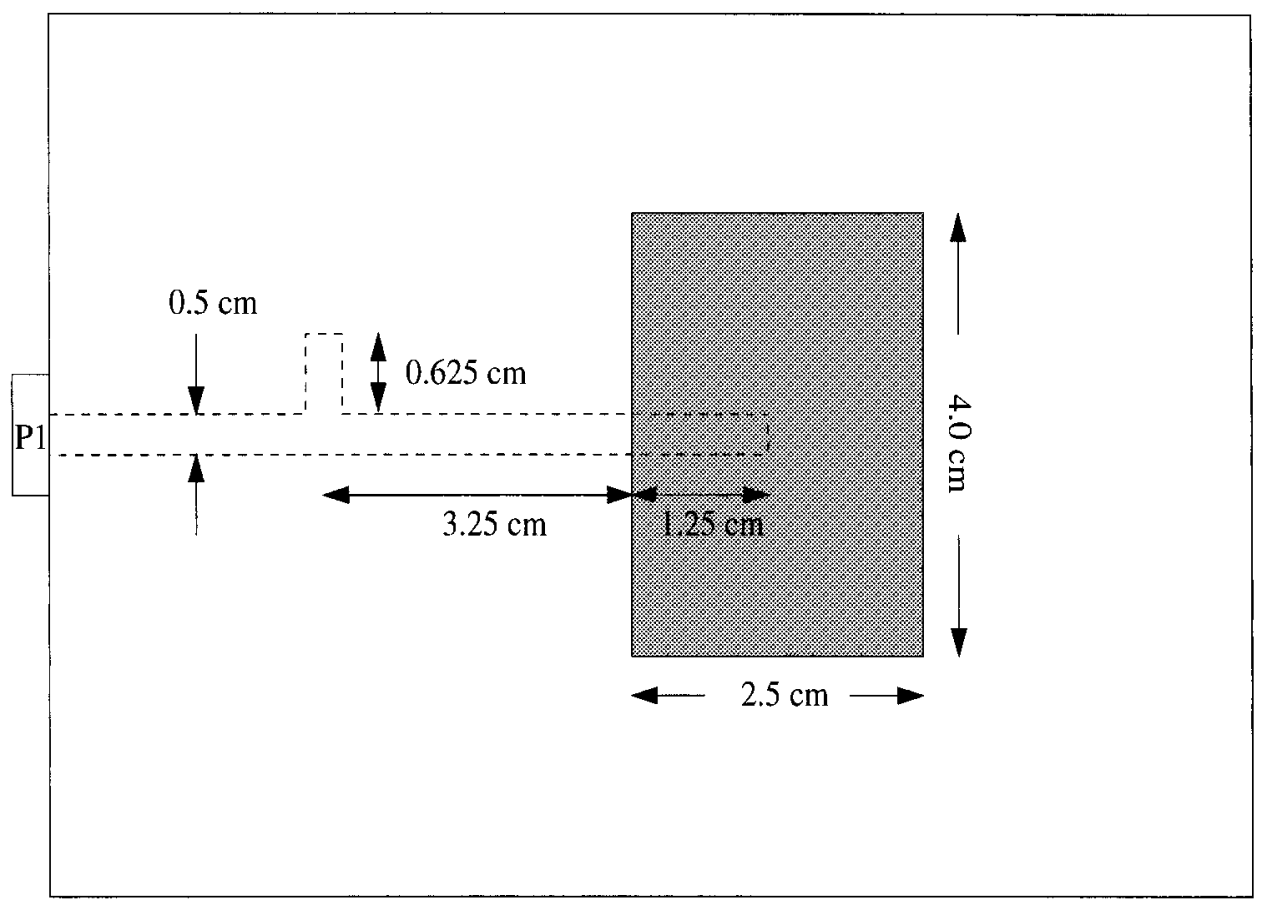

Figure 12. Geometry of the proximity-coupled microstrip antenna showing the input port (not to scale).

terminated in $377 \Omega$, and the free space above the structure should be made slightly lossy. Even then, the results obtained show some artifacts (Fig. 14), and the computation time increases significantly because of the increased number of unknown for the larger box. The results obtained by the approach presented in this article agree quite well with the results obtained from the $\mathrm{em}$ software, except the artifacts, but there is a slight shift in the position of the experimental curve that could be attributed to de-embedding of the experimental data.

Finally, a microstrip line with a shunt-shortcircuited stub (Fig. 16) is analyzed. The thickness of the substrate is $0.02032 \mathrm{~cm}$ (=8 mils), and $\varepsilon_{r}=4$. It should be noted that the use of vias or shorting pins in the em software has some restrictions: (i) the assumption of uniform current distribution along the vertical connection; and (ii) limitation on the length of the vertical conductor

Air

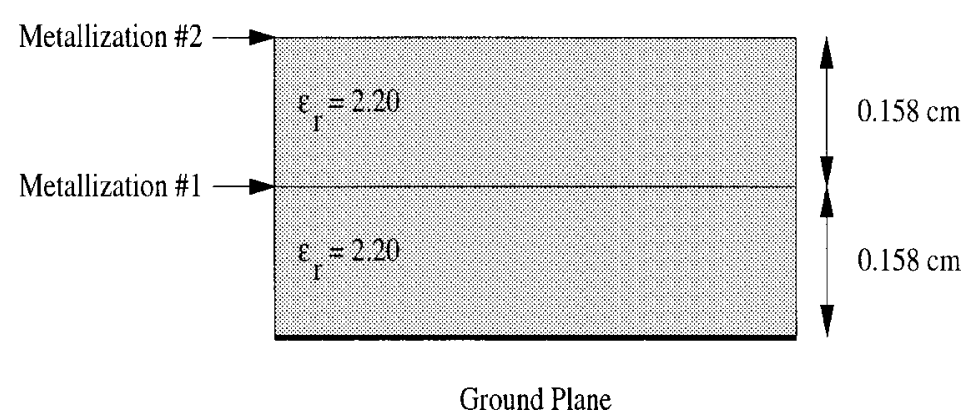

Figure 13. Layer information for the proximity-coupled microstrip antenna shown in Figure 12. 


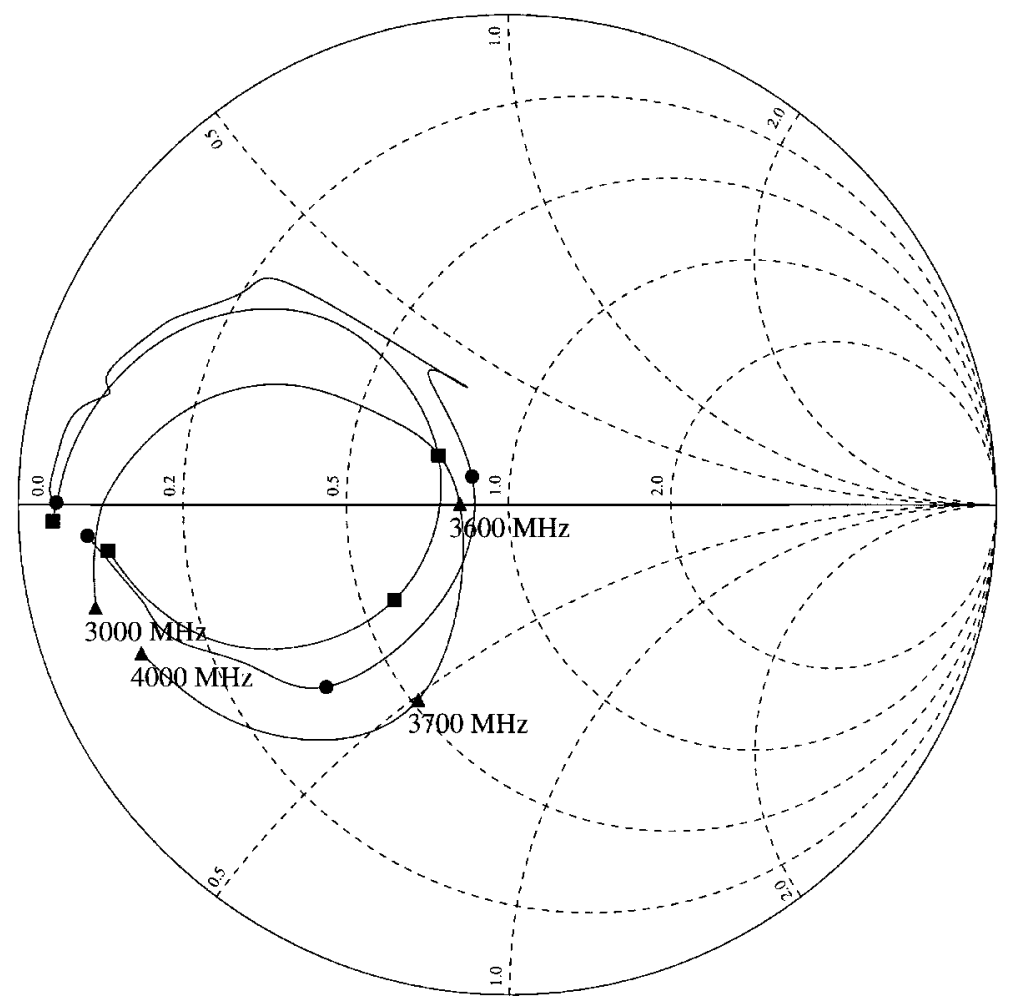

Figure 14. $\Gamma_{\text {in }}$ of the proximity-coupled microstrip antenna shown in Figure 12 without tuning stub (square: MPIE; circle: em; triangle: measured [31]).

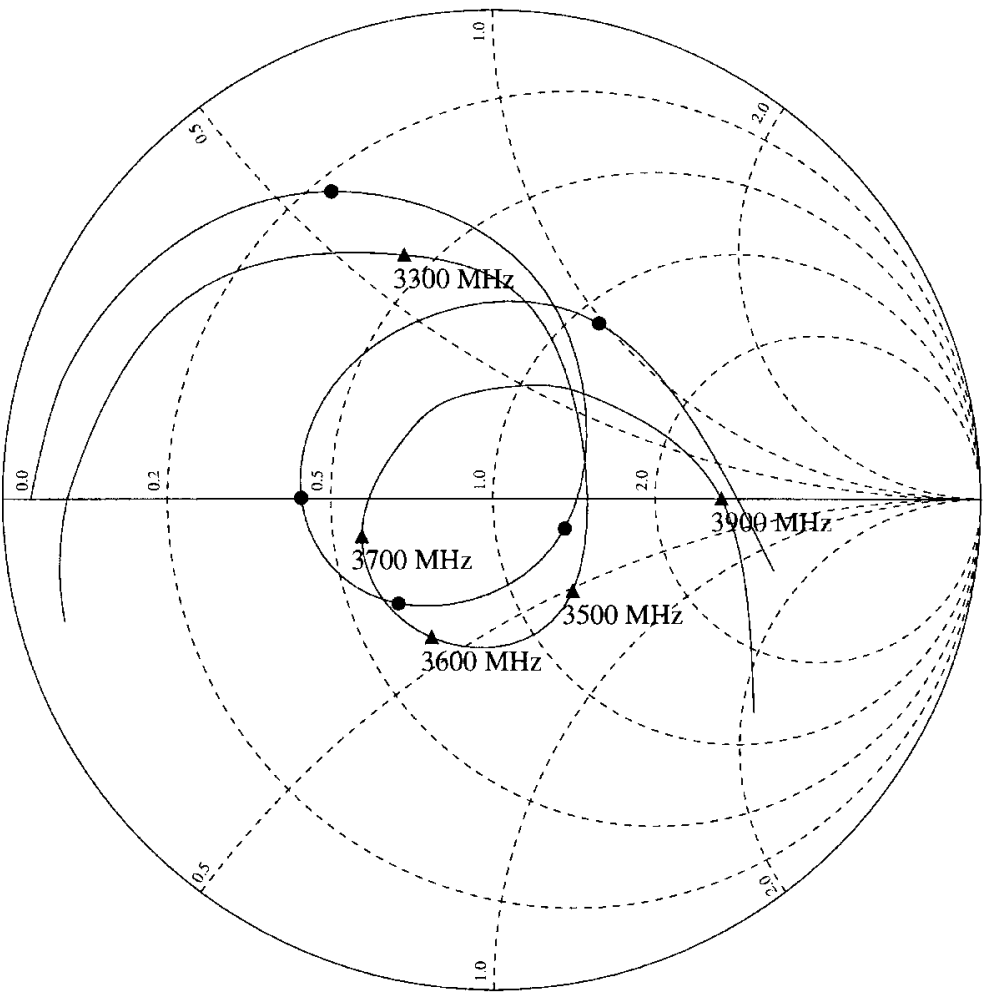

Figure 15. $\Gamma_{i n}$ of the proximity-coupled microstrip antenna shown in Figure 12 with tuning stub (circle: MPIE; triangle: measured [31]). 


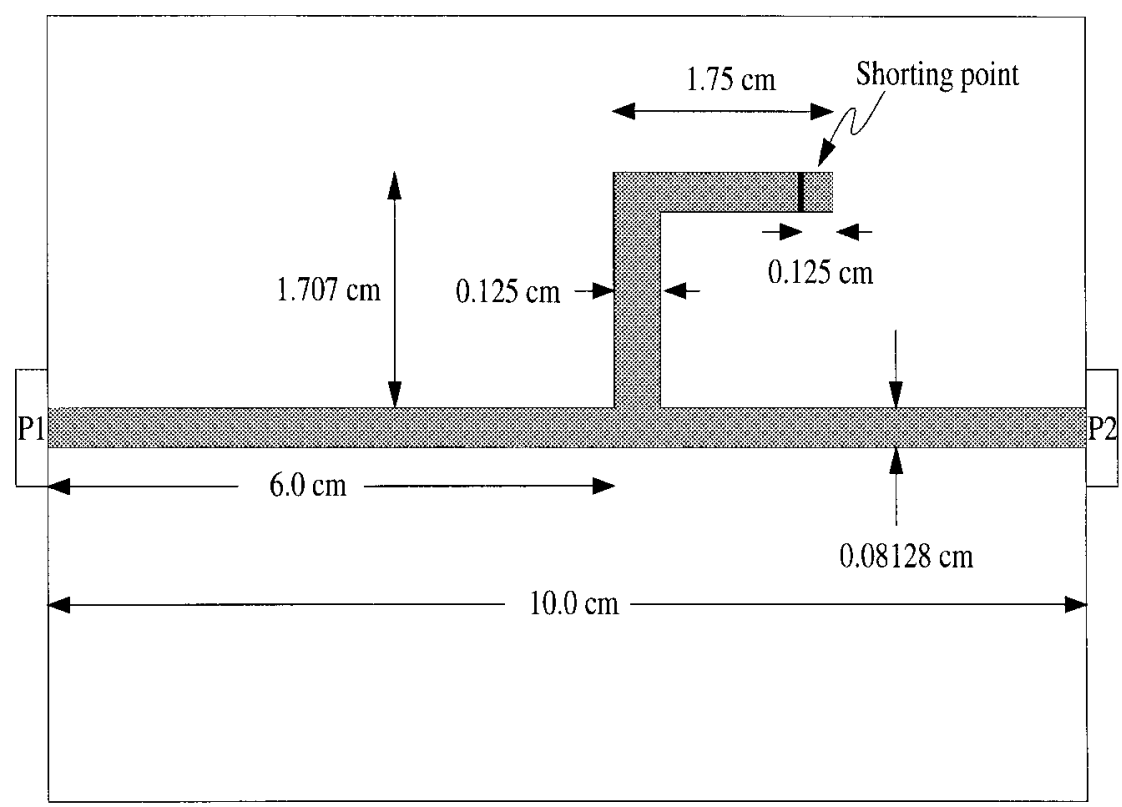

Figure 16. Geometry of a short-circuited microstrip line showing the ports (not to scale).

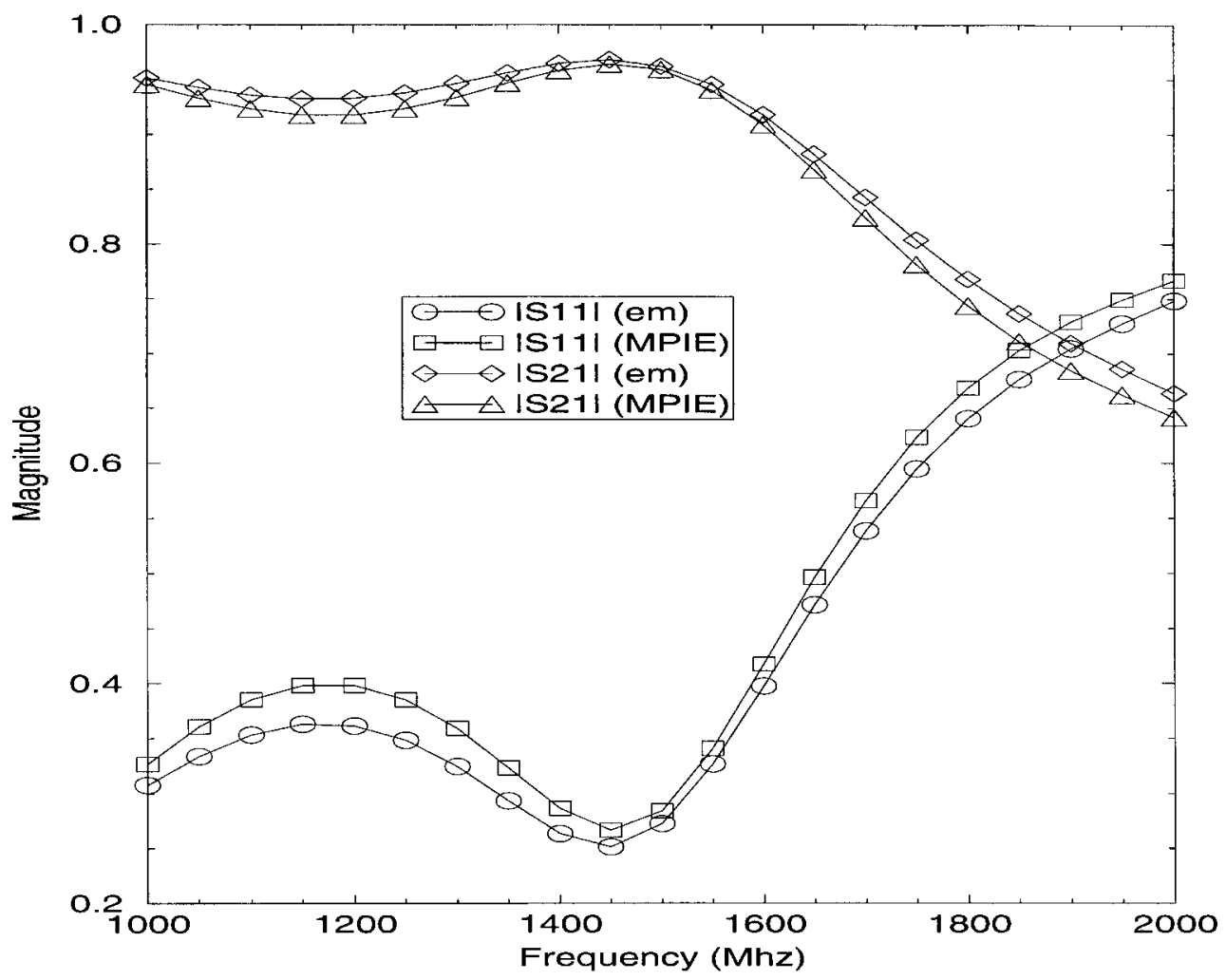

Figure 17. Magnitudes of $S_{11}$ and $S_{21}$ of the microstrip line shown in Figure 16 . 


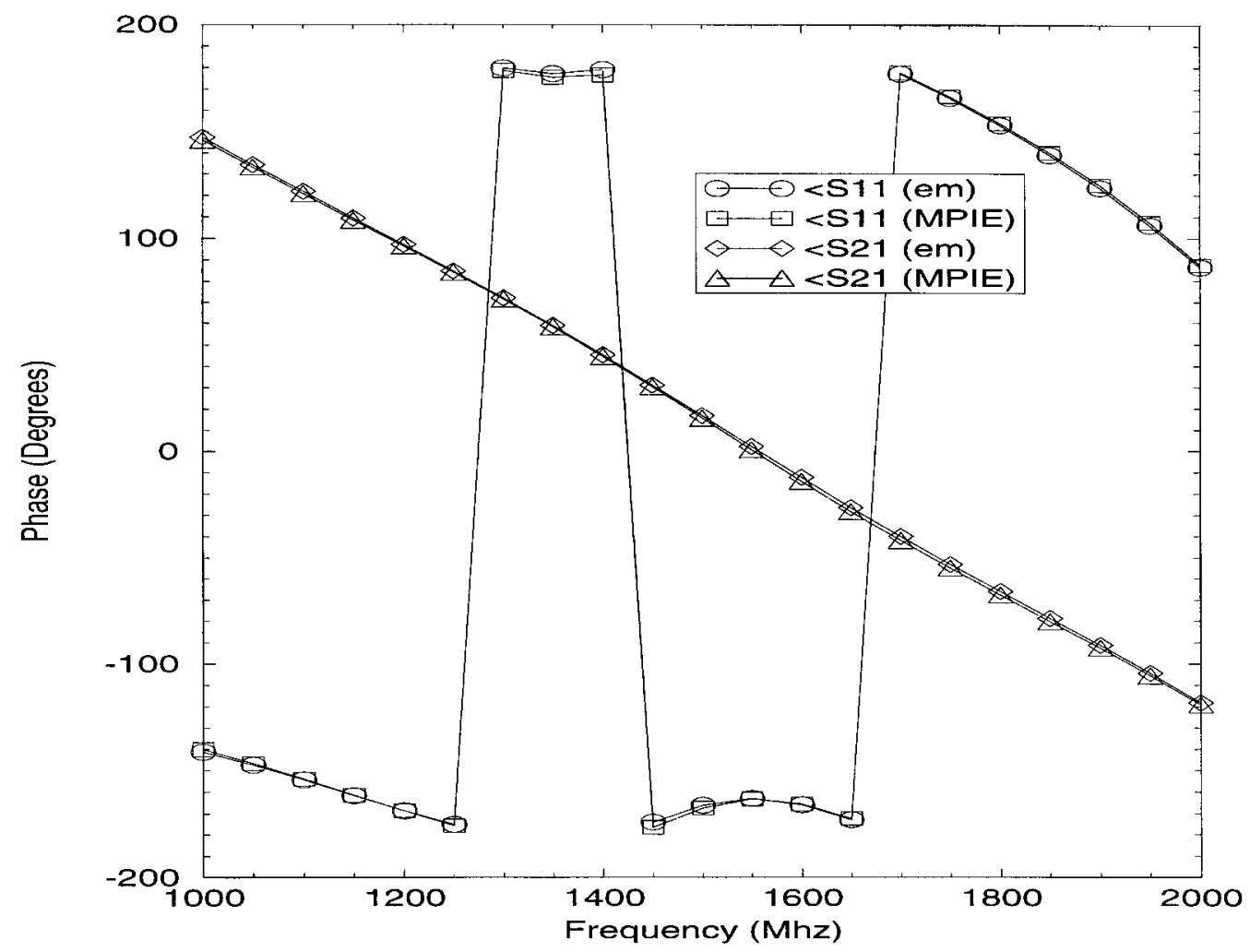

Figure 18. Phases of $S_{11}$ and $S_{21}$ of the microstrip line shown in Figure 16.

(small fraction of a wavelength). On the other hand, the method presented here has no such restrictions, and can handle any length of the vertical conductors with the rooftop basis functions to represent the current. The results obtained from both methods are shown in Figures 17 and 18, and they are in good agreement for this very short shorting pin.

\section{CONCLUSIONS}

In this article, we have presented an efficient and accurate technique for EM simulations of printed geometries in multilayer media. The technique is based on the spatial-domain MoM used with the closed-form Green's functions. It was applied to some printed geometries, and it was demonstrated that the results obtained are in good agreement with the results obtained from a professional electromagnetic analysis software. The advantages of this technique can be stated as follows: (i) it is very suitable for optimization; (ii) it can handle radiating structures efficiently; (iii) the vertical connections with arbitrary lengths can be easily modeled: and (iv) it has the potential of being the most efficient EM software for passive MMIC components.

\section{REFERENCES}

1. P. Silvester and P. Benedek, "Equivalent Capacitance of Microstrip Open Circuits," IEEE Trans. Microwave Theory Tech., Vol. MTT-20, August 1972, pp. 511-516.

2. P. Benedek and P. Silvester, "Equivalent Capacitance of Microstrip Gaps and Steps," IEEE Trans. Microwave Theory Tech., Vol. MTT-20, November 1972, pp. 729-733.

3. P. Silvester and P. Benedek, "Equivalent Discontinuities Capacitances for Right-Angle Bends, Tjunctions, and Crossins," IEEE Trans. Microwave Theory Tech., Vol. MTT-21, May 1973, pp. 341-346.

4. R. F. Harrington, Field Computations by Moment Methods. Macmillan, New York, 1968.

5. J. Jin, The Finite Element Method in Electromagnetics. John Wiley, New York, 1993.

6. K. Kunz and R. Luebber, The Finite Difference Time Domain Method for Electromagnetics. CRC Press, Boca Raton, FL, 1993.

7. J. R. Mosig, “Arbitrarily Shaped Microstrip Structures and Their Analysis with a Mixed Potential 
Integral Equation," IEEE Trans. Microwave Theory Tech., Vol. MTT-36, February 1988, pp. 314-323.

8. I. Park, R. Mittra, and M. I. Aksun, "Numerically Efficient Analysis of Planar Microstrip Configurations Using Closed-form Green's functions," IEEE Trans. Microwave Theory Tech., Vol. 43, February 1995, pp. 394-400.

9. K. A. Michalski and Juan R. Mosig, "Discrete Complex Image MPIE Analysis of Coax-Fed Coupled Vertical Monopoles in Grounded Dielectric Substrate: Two Formulations," IEE Proc. Microwave Ant. Propagat., Vol. 142, June 1995, pp. 269-274.

10. J. R. Mosig and F. E. Gardiol, "Analytical and Numerical Techniques in the Green's Function Treatment of Microstrip Antennas and Scatters," IEE Proc., Vol. 130, March 1983, pp. 175-182.

11. Y. L. Chow, J. J. Yang, D. G. Fang, and G. E. Howard, "A Closed-Form Spatial Green's Function for the Thick Microstrip Substrate," IEEE Trans. Microwave Theory Tech., Vol. 39, March 1991, pp. 588-592.

12. L. Alatan, M. I. Aksun, K. Mahadevan, and T. Birand, "Analytical Evaluation of the MoM Matrix Elements," IEEE Trans. Microwave Theory Tech., Vol. 44, April 1996, pp. 519-525.

13. K. Naishadham and P. Misra, "Order Recursive Gaussian Elimination and Efficient CAD of Microwave Circuits," IEEE MTT-S Dig., 1995, pp. $1435-1438$.

14. K. A. Michalski and Dalian Zheng, "Electromagnetic Scattering and Radiation by Surfaces of Arbitrary Shape in Layered Media, Part I: Theory," IEEE Trans. Ant. Propagat., Vol. AP-38, March 1990, pp. 335-344.

15. A. Sommerfeld, Partial Differential Equations in Physics. Academic Press, New York, 1949.

16. G. Dural and M. I. Aksun, "Closed-Form Green's Functions for General Sources and Stratified Media," IEEE Trans. Microwave Theory Tech., Vol. 43, July 1995, pp. 1545-1552.

17. M. I. Aksun, "A Robust Approach for the Derivation of Closed-Form Green's Functions," IEEE Trans. Microwave Theory Tech., Vol. 44, May 1996, pp. 651-658.

18. Y. Hua and T. K. Sarkar, "Generalized Pencil-ofFunction Method for Extracting Poles of an EM
System from Its Transient Response," IEEE Trans. Ant. Propagat., Vol. AP-37, February 1989, pp. 229-234.

19. W. C. Chew, Waves and Fields in Inhomogeneous Media. Van Nostrand Reinhold, New York, 1990.

20. J. C. Rautio, "A De-Embedding Algorithm for Electromagnetics," Int. J. MIMICAE, Vol. 1, 1991, pp. 282-287.

21. G. V. Eleftheriades and J. R. Mosig, "On the Network Characterization of Planar Passive Circuits Using the Method of Moments," IEEE Trans. Microwave Theory Tech., Vol. 44, March 1996, pp. 438-445.

22. T. K. Sarkar, Z. A. Maricevic, and M. Kahrizi, "An Accurate De-Embedding Procedure for Characterizing Discontinuities," Int. J. MIMICAE, Vol. 2, 1992, pp. 135-143.

23. R. Kipp and C. H. Chan, "Triangular-Domain Basis Functions for Full-Wave Analysis of Microstrip Discontinuities," IEEE Trans. Microwave Theory Tech., Vol. 41, June/July 1993, pp. 1187-194.

24. F. Gardiol, Microstrip Circuits. Wiley, New York, 1994.

25. H. Howe, Jr. Stripline Circuit Design. Artech House. Dedham, MA, 1974.

26. K. C. Gupta, R. Garg, and I. J. Bahl, Microstrip Lines and Slotlines. Artech House, Dedham, MA, 1979.

27. J. C. Rautio, "A New Definition of Characteristic Impedance," IEEE MTT-S Dig., 1991, pp. 761-764.

28. J. Mason Carroll and K. Chang, "Full-Wave Convergence Analysis of Microstrip Transmission Parameters," Int. J. MIMICAE, Vol. 4, April 1994, pp. $140-147$.

29. B. Bhat and S. K. Koul, Stripline-Like Transmission Lines for Microwave Integrated Circuits. Wiley, Chichester, UK, 1989.

30. W. Schwab, F. Boegelsack, and W. Menzel, "Multilayer Suspended Stripline and Coplanar Line Filters," IEEE Trans. Microwave Theory Tech., Vol. MTT-42, July 1994, pp. 1403-1407.

31. D. M. Pozar and B. Kaufman, "Increasing the Bandwidth of a Microstrip Antenna by Proximity Coupling," Electron. Lett., Vol. 23, April 1987, pp. 368-369.

\section{BIOGRAPHIES}

Noyan Kınayman received the BSc and MSc degrees, both in electrical and electronic engineering, from Middle East Technical University (METU), Ankara, Turkey, in 1990 and 1993, respectively. During the period from June 28, 1990 to September 1, 1994, he worked in the Department of Preparation to Production, Aselsan Military Electronics Inc., as a design and test engineer. His major duty was to design computer-controlled and manual RF test systems which are used on the tests of various military and nonmilitary radios. In October 1994, he joined the EE Department of Bilkent University as a research assistant. His major professional interest is numerical solution of electromagnetic problems. (Photo not available.) 
M. I. Aksun received the BS and MS degrees in electrical and electronics engineering from the Middle East Technical University, Ankara, Turkey, in 1981 and 1983, respectively, and the $\mathrm{PhD}$ degree in electrical and computer engineering from the University of Illinois at Urbana-Champaign in 1990. From 1990 to 1992, he was a postdoctoral fellow at the Electromagnetic Communications Laboratory, University of Illinois
Urbana-Champaign. Since 1992, he has been on the faculty of the Department of Electrical and Electronics Engineering at Bilkent University, Ankara, Turkey, where he is currently an associate professor. His research interests include numerical methods for electromagnetics, microstrip antennas, and microwave and millimeter-wave integrated circuits. (Photo not available.) 